\title{
A high-cholesterol diet enriched with polyphenols from Oriental plums (Prunus salicina) improves cognitive function and lowers brain cholesterol levels and neurodegenerative-related protein expression in mice
}

\author{
Ping-Hui Kuo ${ }^{1}$, Ching-I Lin ${ }^{2}$, Yue-Hwa Chen ${ }^{1}$, Wan-Chun Chiu ${ }^{1}$ and Shyh-Hsiang Lin $^{1 *}$ \\ ${ }^{1}$ School of Nutrition and Health Sciences, Taipei Medical University, 250 Wu-Hsing Street, Taipei, Taiwan, ROC \\ ${ }^{2}$ Department of Nutrition and Health Sciences, Kainan University, Taoyuan, Taiwan, ROC \\ (Submitted 9 September 2014 - Final revision received 5 February 2015 - Accepted 12 February 2015 - First published online 13 April 2015)
}

\section{Abstract}

Ageing accompanied by a decline in cognitive performance may be a result of the long-term effects of oxidative stress on neurologic processes. It has been shown that high-cholesterol contents in the blood and brain may lead to the deposition of the $\beta$-amyloid (A $\beta$ ) protein in the brain, which damages brain cells. The present study was designed to observe the effect of polyphenol-rich Oriental plums on cognitive function and cerebral neurodegeneration-related protein expression in mice that were fed a high-cholesterol diet for 5 months. The study consisted of four groups: the control (Ctrl) group, which was fed the American Institute of Nutrition (AIN)-93M diet; the high cholesterol (HC) group, which was fed the AIN-93M diet with 5\% cholesterol; the high cholesterol + low Oriental plum (LOP) group, which was fed the AIN-93M diet with $5 \%$ cholesterol and $2 \%$ Oriental plum powder; and the high cholesterol + high Oriental plum (HOP) group, which was fed the AIN-93M diet with 5\% cholesterol and 5\% Oriental plum powder. Measurements of cognitive function were assessed using the Morris water maze, and the mRNA expression of cholesterol hydroxylase ( $C y p 4 O$ ), $A \beta$ and $\beta$-secretase 1 (BACE1) were analysed. The results showed that cholesterol concentrations in both the blood and the brain were significantly higher in the HC group than in the Ctrl and HOP groups at the end of the trial. The high-cholesterol diet per se produced significant cognitive deficits, which were accompanied by a significantly increased mRNA expression of $C y p 46, B A C E 1, A \beta$ and 24-hydroxycholesterol in the brain cortex and hippocampus. However, all of these variables were non-significantly increased in the HOP group as compared to the Ctrl group. In conclusion, incorporating polyphenol-enriched Oriental plum into a high-cholesterol diet can ameliorate some of the symptoms of neurodegenerative conditions.

Key words: Alzheimer's disease: Cholesterol: Oriental plums: $\beta$-Amyloid: 24-Hydroxycholesterol: Neurodegeneration

As the proportion of the elderly population in the world increases, so will the prevalence of cognitive impairment as a result of normal ageing and neurodegenerative conditions such as Alzheimer's disease (AD) ${ }^{(1)}$, which is one of the most common forms of neurodegenerative disease. Patients who suffer from cognitive deficits exhibit memory loss, delusions and problems carrying out activities associated with normal daily life. The pathologic characteristics of $\mathrm{AD}$ include the presence and accumulation of $\beta$-amyloid (A $\beta$ ), neurofibrillary tangles, inflammation, oxidative stress and cell loss in the brain ${ }^{(2)}$. There are two subtypes of AD. One is caused by genetics (early-onset AD), and the other, more common, subtype is called late-onset AD. Factors that cause late-onset $\mathrm{AD}$ include ageing, diabetes mellitus, hypercholesterolaemia and oxidative stress ${ }^{(3)}$.
Studies have examined the relationship between increased plasma cholesterol concentrations and $\mathrm{AD}^{(4)}$. They have also indicated that the cholesterol level in the brain is one of the factors that regulates amyloid precursor protein (APP) processing and $\mathrm{A} \beta$ formation ${ }^{(5)}$. The accumulation of cholesterol in hippocampal neurons, which results in accelerated cleavage of the APP into amyloidogenic components, has been observed in cell culture results ${ }^{(6)}$. In the brain, cholesterol almost exclusively originates from in situ synthesis, whereas circulating cholesterol is normally prevented from entering the central nervous system by the blood-brain barrier $^{(7)}$. Because cholesterol cannot be eliminated from the central nervous system and may be toxic to neurons when it is present in excess, it is converted to 24-hydroxycholesterol (24-OHC) by 24-hydroxylase (also called Cyp46) before being transported

Abbreviations: 24-OHC, 24-hydroxycholesterol; A $\beta$, $\beta$-amyloid; AD, Alzheimer's disease; AIN, American Institute of Nutrition; APP, amyloid precursor protein; Ctrl, control; Cyp46, 24-hydroxylase; HC, high cholesterol; HOP, high cholesterol + high Oriental plum; LOP, high cholesterol + low Oriental plum.

*Corresponding author: S.-H. Lin, fax +886 22737 3112, email lin5611@tmu.edu.tw 
out of the brain. This mechanism constitutes the major pathway of cholesterol homoeostasis in the brain. Epidemiological studies have indicated that 24-OHC concentrations in cerebrospinal fluid are increased in AD patients ${ }^{(8)}$.

The Oriental plum (Prunus salicina Lindley), also called the Chinese plum or Japanese plum, is rich in polyphenols and anthocyanins. It has been shown that consuming polyphenolrich foods may prevent the onset of $\mathrm{AD}^{(9)}$. In addition, the accumulation of $\mathrm{A} \beta$ in the brain was reduced and cognitive degradation was slowed in streptozotocin-induced diabetic rats that were fed a powered plum diet ${ }^{(10)}$. It has also been shown that blood cholesterol concentrations in rats can be reduced in a dose-response manner by consuming a polyphenol-rich diet ${ }^{(11,12)}$. The purpose of the present study was therefore to examine the effects of consuming Oriental plums on cognitive performance and cerebral neurodegeneration-related protein expression in $\mathrm{C} 57 \mathrm{BL} / 6$ mice that were fed a cholesterol-enriched diet.

\section{Materials and methods}

\section{Animals and diets}

The present experiment was approved by the Institutional Animal Care and Use Committee or Panel (IACUC/IACUP) of Taipei Medical University. A total of sixty 4-week-old male C57BL/6-strain mice were purchased from BioLASCO. Before the experiment began, the mice had access to food and water ad libitum and were housed at $23 \pm 2^{\circ} \mathrm{C}$. After being allowed to acclimatise to these conditions for 2 weeks, the mice were randomly divided into four groups of twenty mice each and kept on a $12 \mathrm{~h}$ light $-12 \mathrm{~h}$ dark cycle. Each group received one of the following treatments for 20 weeks ( 5 months): the control (Ctrl) group was fed the American Institute of Nutrition (AIN)-93M diet; the high cholesterol (HC) group was fed the AIN-93M diet with 5\% cholesterol; the high cholesterol + low Oriental plum (LOP) group was fed the AIN-93M diet with 5\% cholesterol and $2 \%$ Oriental plum powder; and the high cholesterol + high Oriental plum (HOP) group was fed the AIN-93M diet with 5\% cholesterol and 5\% Oriental plum powder. Oriental plums ( $P$. salicina) were purchased from a local market. Dried plum powder was produced by freezedrying and then grinding the plums. The experimental diets were prepared according to the AIN-93M formulation with or without $5 \%$ cholesterol and/or dried plum powder. The highcholesterol diet supplemented with $5 \%$ cholesterol for inducing hypercholesterolaemia in mice was modified from a previous study ${ }^{(13)}$. Maize starch powder in the HC group was partly substituted with dried plum powder in the LOP and HOP groups. The amounts of dried plum powder used in the experimental diets were based on our earlier findings, which showed that Oriental plums had beneficial effects on cognitive function $^{(10)}$. The total phenolic and anthocyanin contents of the dried powder were 24.09 (SEM 0.11) mg gallic acid equivalent/g dried weight of powder and $2 \cdot 28$ (SEM 0.01) $\mathrm{mg}$ cyanidin-3-glucoside equivalent/g dried weight of powder, respectively. The measurements of the antioxidant contents of the dried plum power were described in our previous work ${ }^{(10)}$.
Diet compositions are shown in Table 1. All mice consumed food and water ad libitum for 20 weeks before cognitive testing was conducted. The body weights of the mice were measured every week. Blood samples were collected every month to measure thiobarbituric acid-reactive substances and cholesterol concentrations. After 5 months of treatment, the mice were starved for $12 \mathrm{~h}$ and then killed using anaesthesia with an intraperitoneal injection of a solution of Zoletil 50 (Virbac) and $2 \%$ Rompun (1:1 ratio, $1 \mathrm{ml} / \mathrm{kg}$ body weight; Bayer). The mice were then transcardially perfused with ice-cold $0 \cdot 1 \mathrm{M}$-PBS $(\mathrm{pH} 7 \cdot 4)$, and their brains were rapidly removed. The cortices and hippocampi were immediately collected, snap-frozen in liquid $\mathrm{N}_{2}$ and stored at $-80^{\circ} \mathrm{C}$ until analysis.

\section{Morris water maze task}

The Morris water maze task has been used extensively in investigations related to cognitive function in rodents. In the present study, it was performed after 5 months of treatment according to the method described by Vorhees \& Williams ${ }^{(14)}$. The apparatus (TSE Systems) consisted of a circular pool (diameter: $150 \mathrm{~cm}$, height: $100 \mathrm{~cm}$ ) filled with water (depth: $60 \mathrm{~cm}, 24 \pm 2^{\circ} \mathrm{C}$ ) that was placed in a room that had consistently located spatial cues. An escape platform (diameter: $14 \mathrm{~cm}$ ) was placed in the middle of one of the quadrants $1 \mathrm{~cm}$ below the water surface and equidistant from the side wall and the middle of the pool. The platform provided the only escape from the water and was located in the same quadrant in every trial. Four different starting positions were equally spaced along the perimeter of the pool. On each training day during the acquisition trial, all four starting positions were used one time in a random sequence (i.e. four training trials per day). The trial began by placing the animal in the water facing the wall of the pool at one of the starting points. If the mouse failed to escape within $60 \mathrm{~s}$, it was gently conducted to the platform. It was allowed to stay on the platform for $15 \mathrm{~s}$. The inter-trial interval was $15 \mathrm{~min}$. After each trial, the mice were dried, and they were returned to their cages at the end of the session. The animals were trained for $3 \mathrm{~d}$. At $24 \mathrm{~h}$ after the last training session, the mice were submitted to a probe trial. Before this session, the submerged platform was removed. The retention test consisted of placing the mice in the water for $60 \mathrm{~s}$. Performances

Table 1. Diet composition of each group

\begin{tabular}{lrrrr}
\hline Ingredient (\%) & Ctrl & HC & LOP & HOP \\
\hline Maize starch & 46.8 & 44.6 & $42 \cdot 6$ & 39.2 \\
Dextrinised maize starch & 15.6 & 14.8 & 14.8 & 15.0 \\
Casein & 14.1 & 13.4 & 13.4 & 13.5 \\
Sucrose & 10.0 & 9.6 & 9.6 & 9.7 \\
Cellulose & 5.0 & 4.8 & 4.8 & 4.8 \\
Soyabean oil & 4.0 & 3.8 & 3.8 & 3.9 \\
Mineral mixture & 3.5 & 3.3 & 3.3 & 3.4 \\
Vitamin mixture & 1.0 & 1.0 & 1.0 & 1.0 \\
Cholesterol & 0.0 & 4.8 & 4.8 & 4.8 \\
Plum powder & 0.0 & 0.0 & 1.9 & 4.8 \\
\hline
\end{tabular}

Ctrl, control; HC, high cholesterol; LOP, high cholesterol + low Oriental plum; HOP, high cholesterol + high Oriental plum. 


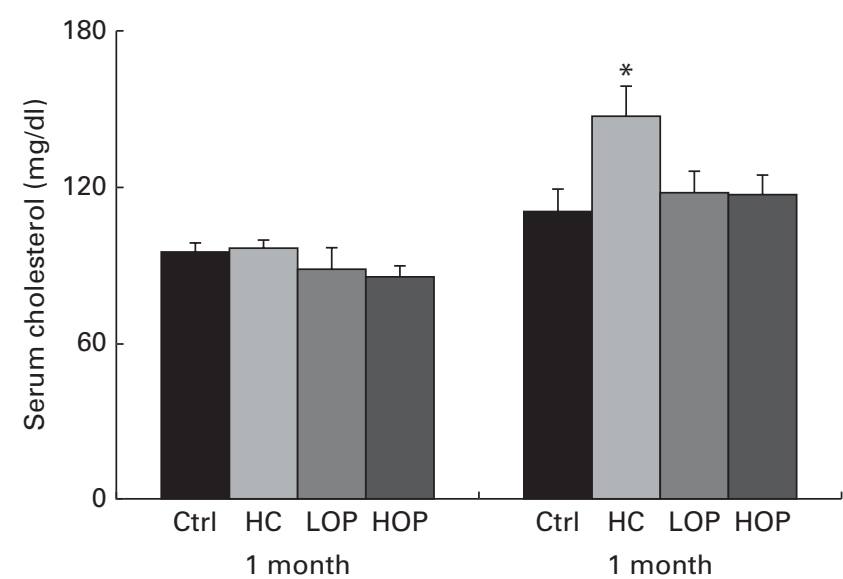

Fig. 1. Serum cholesterol concentrations at 1 month and 5 month during the experimental period. Values are means ( $n 20$ at 0 months; $n 15$ at 5 months), with their standard errors represented by vertical bars. * Mean values were significantly different $(P=0.029$; Duncan post hoc analysis). Ctrl, control; HC, high cholesterol; LOP, high cholesterol + low Oriental plum; HOP, high cholesterol + high Oriental plum. To convert cholesterol from $\mathrm{mg} / \mathrm{dl}$ to $\mathrm{mmol} / \mathrm{l}$, multiply by 0.0258 .

were evaluated and analysed with image tracking software (FG34PATH; HaSoTec), which measured the number of crossings over the original platform location, the latency of arrival at the original platform location and the time spent in the target quadrant.

\section{Serum cholesterol concentrations}

Serum was separated from blood samples by centrifugation at $3000 \mathrm{~g}$ for $10 \mathrm{~min}$ at $4^{\circ} \mathrm{C}$. Concentrations of total cholesterol were determined by the colorimetric enzymatic method using an autoanalyser (Roche Modular P800).

\section{Serum thiobarbituric acid-reactive substances}

A method modified from that described by Yagi ${ }^{(15)}$ was used. Serum $(100 \mu \mathrm{l})$ was reacted with $800 \mu \mathrm{l}$ of $0 \cdot 22 \% \mathrm{H}_{2} \mathrm{SO}_{4}$, $100 \mu \mathrm{l}$ of $10 \%$ phototungstic acid and $200 \mu \mathrm{l}$ of a $0.61 \%$ thiobarbituric acid solution. The samples were heated to $95^{\circ} \mathrm{C}$ for $1 \mathrm{~h}$ before the addition of $400 \mu \mathrm{l}$ of 1 -butanol. The samples were then vigorously stirred for $2 \mathrm{~min}$ and centrifuged at $800 \boldsymbol{g}$ for $15 \mathrm{~min}$. The absorbance of the organic phase was measured at excitation of $515 \mathrm{~nm}$ and emission of $555 \mathrm{~nm}$, and the samples were compared to a blank.

\section{Brain cholesterol and 24-hydroxycholesterol concentrations}

After the Morris water maze test, the mice were killed. The cortices and hippocampi of the mice were excised and minced. Cholesterol was extracted using the procedure described by Folch et al. ${ }^{(16)}$. Total cholesterol was determined using a commercial kit (Roche). Brain 24-OHC content was determined using a 24(S)-hydroxycholesterol ELISA kit (Enzo Life Sciences). The resulting yellow colour was read at $450 \mathrm{~nm}$. The signal was inversely proportional to the concentration of $24-\mathrm{OHC}$ in the sample.

\section{Cyp46 mRNA analysis}

Total RNA was isolated from the cortical and hippocampal samples using TRIzol reagent according to the manufacturer's protocol (Life Technologies). Complementary DNA was synthesised by reverse transcription using $3 \mu \mathrm{g}$ of total RNA. Complementary DNA was synthesised using $1 \mu \mathrm{g}$ of oligo $\mathrm{dT}, 1 \mu \mathrm{l}$ of an RNase inhibitor, $2 \mu \mathrm{l}$ of $10 \mathrm{~mm}$-dNTP mixture, $4 \mu \mathrm{l}$ of $5 \times$ reaction buffer and $1 \mu \mathrm{l}$ of Moloney murine leukaemia virus (MMLV) (RT) in an $8-\mu l$ reaction mixture. After allowing the oligo dT to anneal further at $65^{\circ} \mathrm{C}$ for $5 \mathrm{~min}$, reverse transcription was carried out at $45^{\circ} \mathrm{C}$ for $60 \mathrm{~min}$. The enzyme, RT, was heat-inactivated at $70^{\circ} \mathrm{C}$ for $5 \mathrm{~min}$. To quantify the mouse Cyp46 mRNA, $1-3 \mu \mathrm{g}$ of complementary DNA was used as a temperate for a real-time PCR analysis based on SYBR Green I with the LightCycler ${ }^{\circledR}$ Carousel-Based System (Roche Diagnostics). A 285-bp PCR product containing Cyp46 was amplified using specific primers (forward primer: 5'-AAC TTT GTC ACC TTC TTC ATT GC-3'; reverse primer: 5'-CCA TCA CTG TGA ATG CCA GA-3'). Amplification and detection were done on a LightCycler 480 System (Roche Diagnostics) under the following conditions: an initial $5 \mathrm{~min}$ denaturation step at $94^{\circ} \mathrm{C}$, followed by thirty-five cycles of denaturation at $94^{\circ} \mathrm{C}$ for $10 \mathrm{~s}$, annealing at $60^{\circ} \mathrm{C}$ for $5 \mathrm{~s}$ and extension at $72^{\circ} \mathrm{C}$ for $8 \mathrm{~s}$. The specificity of the amplified PCR product was inspected by performing a melting curve analysis on the LightCycler 480 System. The gene expression of Cyp46 was calculated based on crossing point values obtained by the second derivative maximum procedure (with $\beta$-actin as the housekeeping gene).

\section{$\beta$-Secretase 1 and $\beta$-amyloid protein analysis}

Proteins were extracted from cortical and hippocampal samples using radioimmunoprecipitation assay (RIPA) lysis buffer (50 mm-Tris- $\mathrm{HCl}$ at $\mathrm{pH} 7 \cdot 6,150 \mathrm{~mm}-\mathrm{NaCl}, 0 \cdot 1 \%$ SDS, $0.5 \%$ sodium deoxycholate and $1 \%$ Triton X-100) with protease and a phosphatase inhibitor cocktail (Thermo Fisher Scientific). Extracted proteins were separated by $12 \%$ SDSPAGE. Following electrophoresis, the separated proteins in

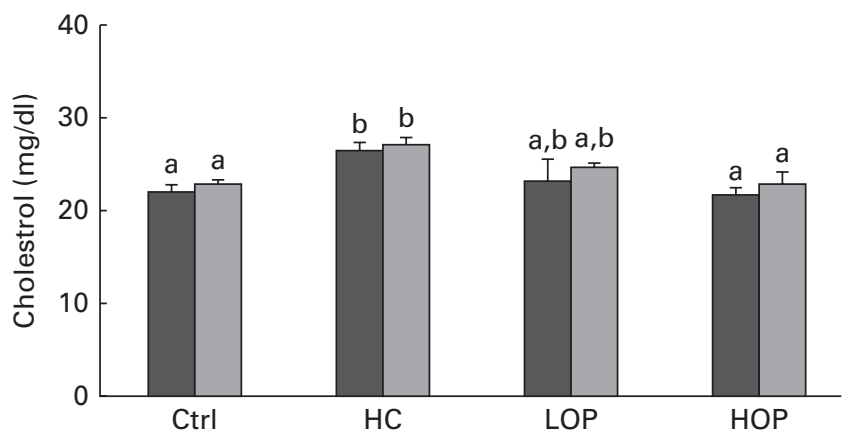

Fig. 2. Cholesterol concentrations in the hippocampus $(\square)$ and cortex ( $\square$ ) at the end of month 5 . Values are means ( $n$ 6-8 per group), with their standard errors represented by vertical bars. ${ }^{a, b}$ Mean values with unlike letters were significantly different from the matched tissue $(P=0.008$; Duncan post hoc analysis). Ctrl, control; HC, high cholesterol; LOP, high cholesterol + low Oriental plum; HOP, high cholesterol + high Oriental plum. To convert cholesterol from $\mathrm{mg} / \mathrm{dl}$ to $\mathrm{mmol} / \mathrm{l}$, multiply by 0.0258 . 

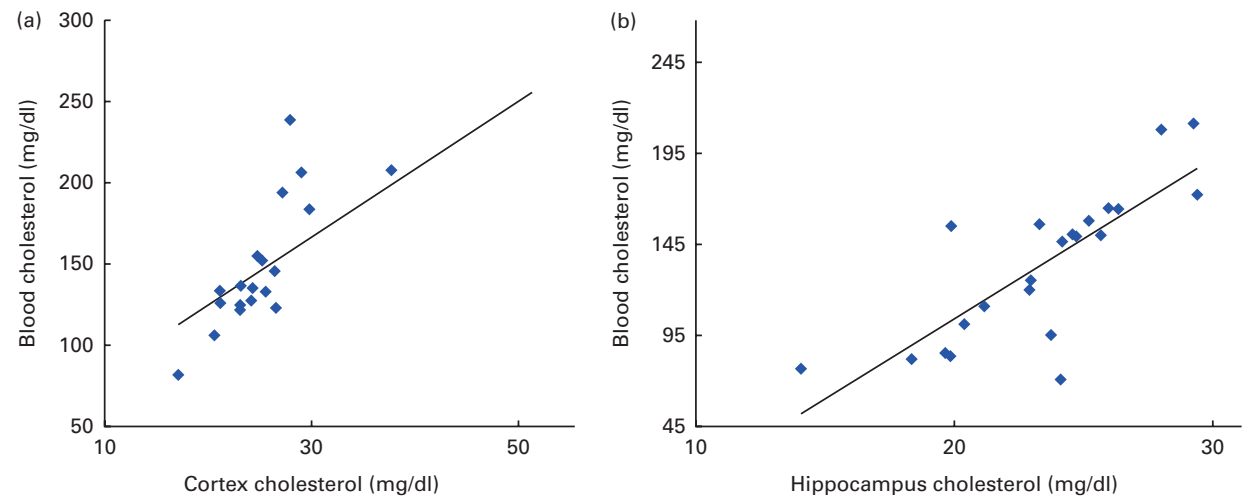

Fig. 3. Correlation between serum and brain cholesterol levels in mice ( $n$ 4-6 per group). (A) Cortex: $r 0.73, P=0.0001 ;$ (B) hippocampus: $r 0.79, P=0.0001$. To convert cholesterol from $\mathrm{mg} / \mathrm{dl}$ to $\mathrm{mmol} / \mathrm{l}$, multiply by 0.0258 . A colour version of this figure can be found online at http://www.journals.cambridge.org/bjn

the gel were transferred onto polyvinylidene difluoride membranes (Millipore) at $80 \mathrm{~mA}$ for $2 \mathrm{~h}$. Once the transfer was complete, the membranes were immediately incubated in blocking buffer for $1 \mathrm{~h}$. To detect the target proteins, blotted membranes were incubated overnight at $4^{\circ} \mathrm{C}$ with primary antibodies against $A \beta_{1-42}$ (mice anti-A $\beta$; Millipore), BACE1 (rabbit anti-BACE; Abcam) and secondary antibodies (goat anti-mouse IgG and goat anti-rabbit IgG (Sigma). Before quantifying the expression of the target proteins, a peroxidase-based detection of the blot was performed with a Western blotting luminal reagent and was visualised with a Biospectrum AC imaging system (UVP). Quantification of the target proteins was analysed using Image-Pro Plus software (Media Cybernetics).

\section{Statistical analysis}

Data are expressed as means with their standard errors. Statistical analyses were performed using SAS version 9.4 (SAS Institute, Inc.) and SPSS version 18.0 (SPSS, Inc.). The correlation between serum and brain cholesterol concentrations was analysed by Pearson's correlation coefficient analysis. A repeated measures ANOVA was performed on the Morris water maze test data. A one-way ANOVA followed by Duncan's post hoc test was performed on the other analyses. Statistical significance was set at $P<0.05$ for all of the tests.

\section{Results \\ Body weight changes and serum cholesterol concentrations}

Mean body weight among the groups did not differ significantly throughout the 5-month experiment period (averages of 23-36g in each group, data not shown). Fig. 1 shows that the mice that consuming a 5\% high-cholesterol diet (the HC group) exhibited a significantly higher concentration in serum cholesterol at the end of the experiment than the Ctrl and HOP groups did $(P=0.029)$. No significant differences in cholesterol concentrations were found among the Ctrl, LOP and HOP groups after 5 months of treatment.

\section{Cortical and hippocampal cholesterol concentrations}

After five months of consuming a $5 \%$ high-cholesterol diet, the HC group had significantly higher cholesterol concentrations $(P=0 \cdot 008)$ in the cortex and hippocampus than the other groups did (Fig. 2). The cholesterol concentration in the cortex and hippocampus in the HOP group was not significantly different from that in the Ctrl group at the end of the experiment.

\section{Correlation between serum and brain cholesterol concentrations in mice}

Fig. 3 shows a significantly positive correlation between serum and cortical (Fig. 3(A), $r 0.73, P=0.0001$ )/hippocampal (Fig. 3(B), $r$ 0.79, $P=0.0001$ ) cholesterol concentrations in the mice from all of the groups.

\section{Lipid peroxidation in serum}

The results of measuring lipid peroxidation in the serum, which is expressed as thiobarbituric acid-reactive substances, showed that the thiobarbituric acid-reactive substances

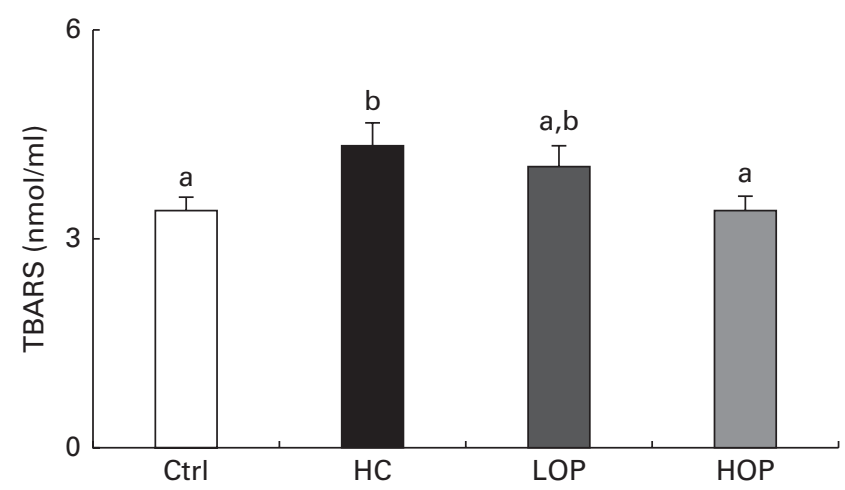

Fig. 4. Serum thiobarbituric acid-reactive substance (TBARS) concentrations in mice. Values are means (n 11-13 per group), with their standard errors represented by vertical bars. ${ }^{\mathrm{a}, \mathrm{b}}$ Mean values with unlike letters were significantly different $(P=0.051$; Duncan post hoc analysis). Ctrl, control; $\mathrm{HC}$, high cholesterol; LOP, high cholesterol + low Oriental plum; HOP, high cholesterol + high Oriental plum. 

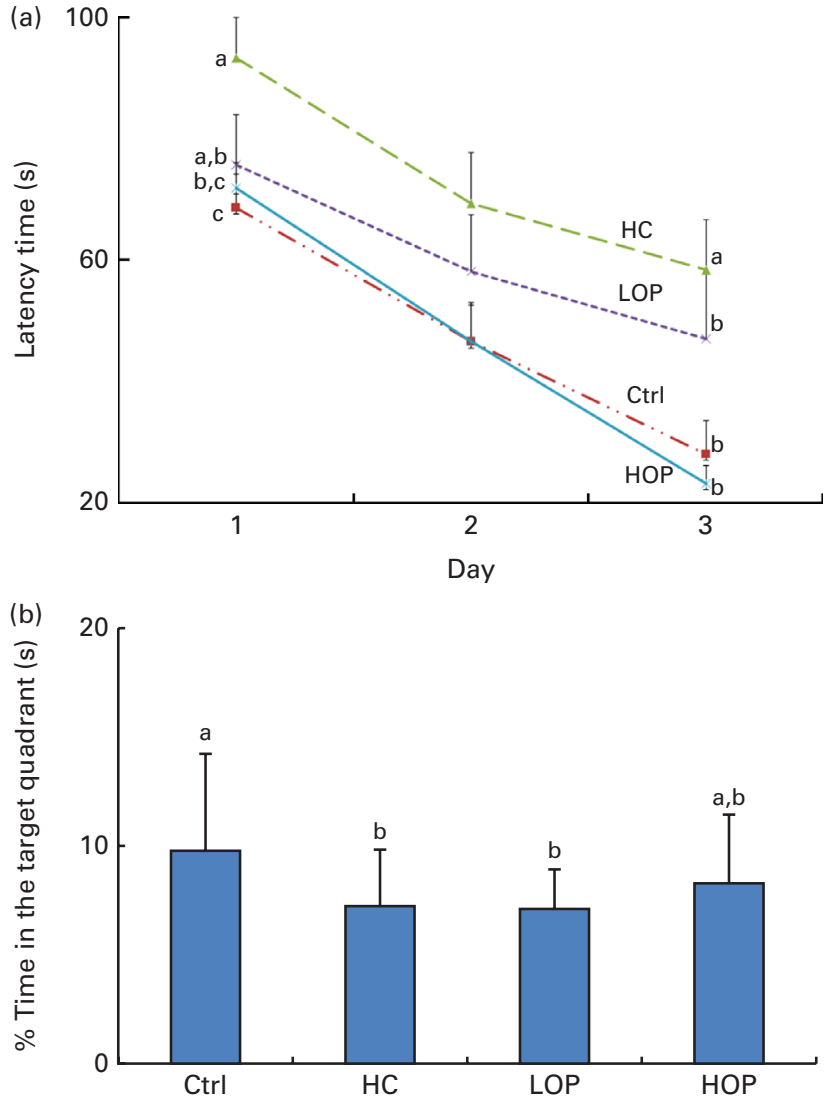

Fig. 5. Cognitive function of mice as assessed by the Morris water maze at month 5. Values are means ( $n 6-8$ per group), with their standard errors represented by vertical bars. (A) Spatial learning and memory trial (latency time to find a hidden platform during a $3 \mathrm{~d}$ acquisition session in four trial blocks). (B) Spatial memory trial (\% time in the target quadrant in the probe trial). ${ }_{a, b, c}$ Mean values with unlike letters were significantly different $(P=0.006$ for day 1; $P=0.0012$ for day 3; $P=0.054$ for day 4; Duncan post hoc analysis). Ctrl, control; HC, high cholesterol; LOP, high cholesterol + low Oriental plum; HOP, high cholesterol + high Oriental plum. A colour version of this figure can be found online at http://www.journals.cambridge.org/bjn

concentration in the serum of the HOP group was significantly lower $(P=0.051)$ than that of the HC group and was not significantly different from that of the Ctrl group at the end of the experiment (Fig. 4)

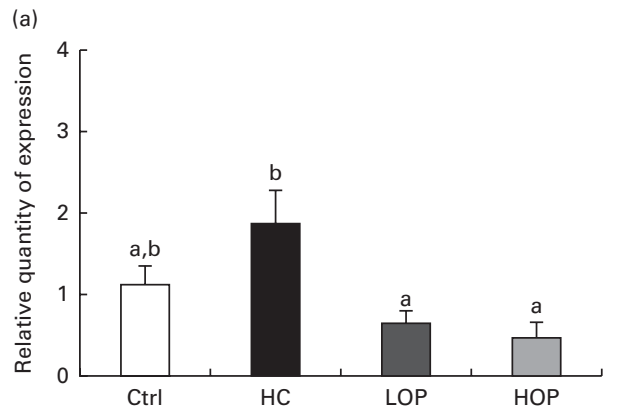

\section{Cognition analysis with the Morris water maze}

Fig. 5(A) shows that after consuming a high-cholesterol diet for 5 months, on day 1 of the task, mice in the HC group had a longer escape latency than those in the Ctrl and HOP groups $(P=0.006)$. On day 3 , mice in the HOP, LOP and Ctrl groups spent significantly shorter time finding the target than those in the HC group did $(P=0.0012)$. On the other hand, on day 4 (Fig. 5(B)), the time spent in the previously learned target quadrant by the HOP group tended to be closer to that of the Ctrl group and longer than those of the HC and LOP groups $(P=0 \cdot 054)$.

\section{Expression of Cyp46 mRNA and 24-hydroxycholesterol concentrations in the brain}

Cyp46 plays an important role in the elimination of brain cholesterol and the conversion of brain cholesterol into 24-OHC. Fig. 6(A) and (B) shows the expression of Cyp46 mRNA in the cortex and hippocampus at the end of the experiment. In the cortex, the expression of Cyp46 mRNA in the HOP and LOP groups was lower than that of the HC group $(P=0.05)$. The concentration of $24-\mathrm{OHC}$ in the cortex and hippocampus was also lower in the HOP group than that of the HC group $(P=0.05)$ (Fig. 7).

\section{Expression of $\beta$-secretase 1 and $\beta$-amyloid proteins in the brains of mice}

$B A C E 1$ and $A \beta$ expressions in the brains of the mice in each group are shown in Figs. 8 and 9. In the cortex and hippocampus, the expression of both $B A C E 1$ and $A \beta$ in the HOP group was significantly lower than that of the HC group $(P=0.05)$.

\section{Discussion}

In the present study, we investigated the protective effect of polyphenol-rich Oriental plums on cognitive function and cerebral neurodegeneration-related protein expression in mice that were fed a high-cholesterol diet. A loss of shortterm memory is commonly observed among AD patients ${ }^{(2)}$. We found that mice that consumed a high-cholesterol diet

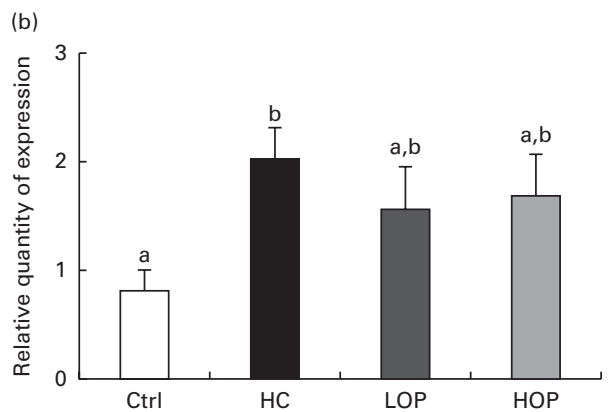

Fig. 6. Expression of 24-hydroxylase (Cyp46) mRNA in (A) the cortex and (B) the hippocampus in mice. Values are means ( $n 6-8$ per group), with their standard errors represented by vertical bars. Total RNA was extracted from the brains of mice and quantified in real time. ${ }^{a, b}$ Mean values with unlike letters were significantly different $(P=0.05$; Duncan post hoc analysis). Ctrl, control; HC, high cholesterol; LOP, high cholesterol + low Oriental plum; HOP, high cholesterol + high Oriental plum. 

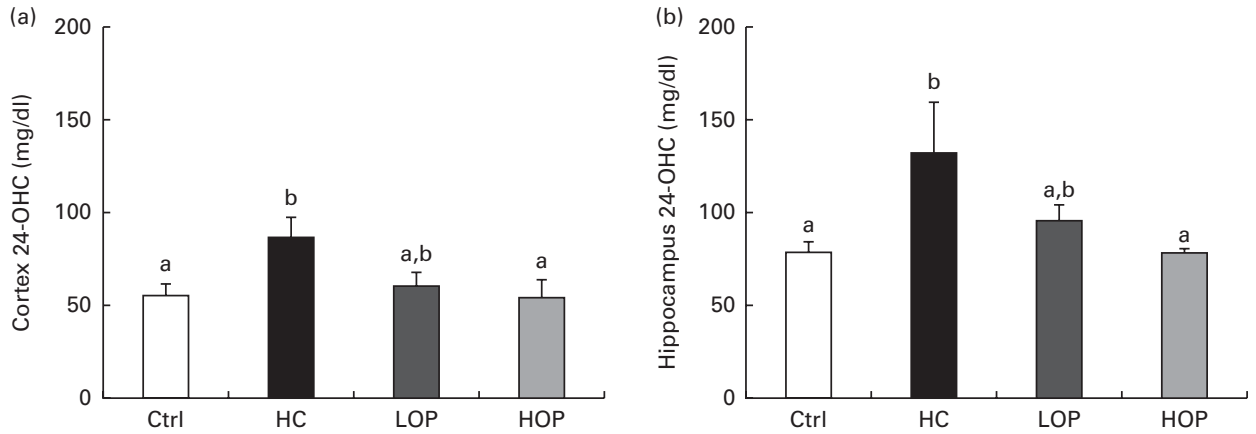

Fig. 7. 24-Hydroxycholesterol (24-OHC) contents in (A) the cortex and (B) the hippocampus in mice. Values are means ( $n$ 6-8 per group), with their standard errors represented by vertical bars. ${ }^{a, b}$ Mean values with unlike letters were significantly different $(P=0.05)$. Ctrl, control; HC, high cholesterol; LOP, high cholesterol + low Oriental plum; HOP, high cholesterol + high Oriental plum. To convert $24-\mathrm{OHC}$ from mg/dl to mmol/l, multiply by 0.0248 .

for a fairly long experimental period ( 5 months) had elevated cholesterol concentrations in the serum and brain as well as elevated levels of $\mathrm{A} \beta$ in the brain when the diet was not supplemented with Oriental plum powder. The accumulation of $\mathrm{A} \beta$ is one of the pathological features of $\mathrm{AD}$. Evidence from earlier studies has shown that a high-cholesterol diet induces oxidative stress and inflammatory response and might be related to neurodegeneration and cognitive deficits ${ }^{(17)}$. Recent findings have indicated that consuming a diet high in cholesterol causes some cognitive decline in mice. In the present study, the addition of 2 and $5 \%$ Oriental plum powder to a high-cholesterol diet ameliorated the increased cholesterol concentrations in mice. In addition, we also found that there was a positive correlation between serum and brain cholesterol concentrations, which indicates that the blood-brain barrier function might be impaired under a long-term high plasma cholesterol concentration and that cholesterol levels in the brain are thus affected.

The pathogenesis of cognitive deficits caused by elevated cholesterol levels in the brain is still not completely understood $^{(18)}$. However, factors such as increased BACE1, 24-OHC and $A \beta$ levels in the brain have been found to be associated with neurodegeneration ${ }^{(8)}$. The $A \beta$ protein is derived from cleaving APP by BACE1 through the $\beta$ pathway. BACE1 is located in cholesterol-rich regions (i.e. in lipid rafts). Thus, elevated cholesterol concentrations may increase the size of lipid rafts and the activity of $\mathrm{BACE} 1^{(1)}$. Some clinical observations provided in vivo and in vitro evidence indicating that cholesterol plays a role in APP processing and A $\beta$ generation $^{(19)}$. It has also been shown that when rabbits were fed a diet enriched with cholesterol, they had increased levels of A $\beta$ in the brain ${ }^{(20)}$. In transgenic mice that expressed a mutant human APP, A $\beta$ deposits increased in the brain along with plasma cholesterol concentrations ${ }^{(21)}$. In the present study, expressions of $B A C E 1$ and $A \beta$ in the HC group were significantly higher than those in the Ctrl and HOP groups, which suggests that cognitive declines in the $\mathrm{HC}$ group may have been caused by higher BACE1 and $\mathrm{A} \beta$ levels.

The conversion of cholesterol into 24-OHC by Cyp46 appears to be one of the most important mechanisms in the central nervous system for transporting cholesterol out of the brain ${ }^{(7)}$. The present results showed that Cyp46 mRNA expression significantly increased in the cortices and hippocampi of the mice in the HC group. Its expression at transcriptional level was shown to be stable and not affected by steroid hormones or other factors, such as statins, cholesterol or oxysterols, but it can be regulated by oxidative stress $^{(22)}$. Thus, the present findings suggest that the increased Cyp46 mRNA expression in cholesterol-treated mice may be a consequence of oxidative stress, which is indicative of the cholesterol accumulation in the brain ${ }^{(23)}$. In the cortex but not the hippocampus, the elevations were significantly blunted or ameliorated when compared with data from the LOP and HOP groups, which suggests that OP might act as
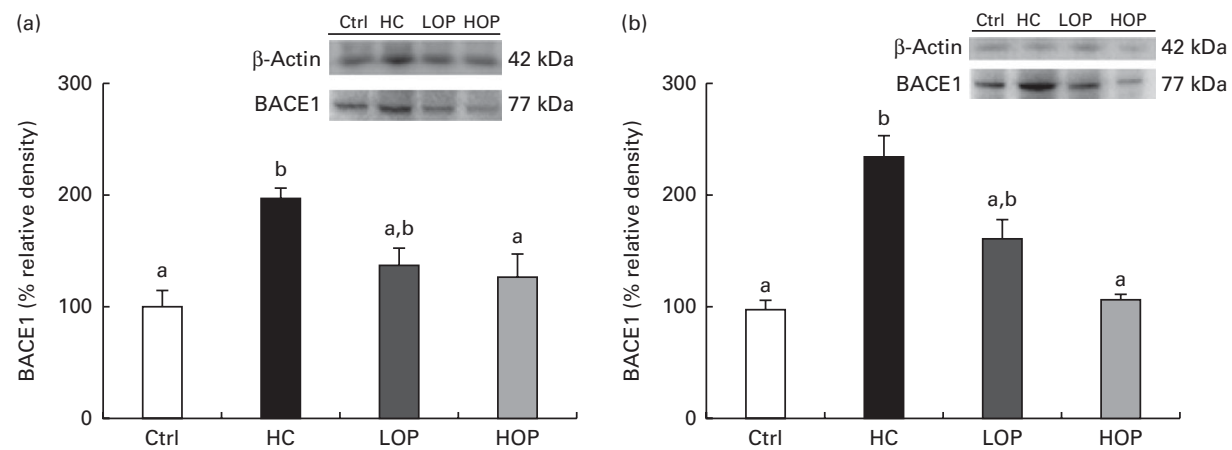

Fig. 8. Expression of the $\beta$-secretase 1 (BACE1) protein in (A) the cortex and (B) the hippocampus in mice. Values are means ( $n 3$ per group) of the percent relative density (with the control set to $100 \%$ ), with their standard errors represented by vertical bars. ${ }^{a, b}$ Mean values with unlike letters were significantly different $(P=0.05$; Duncan post hoc analysis). Ctrl, control; HC, high cholesterol; LOP, high cholesterol + low Oriental plum; HOP, high cholesterol + high Oriental plum. 
(a)

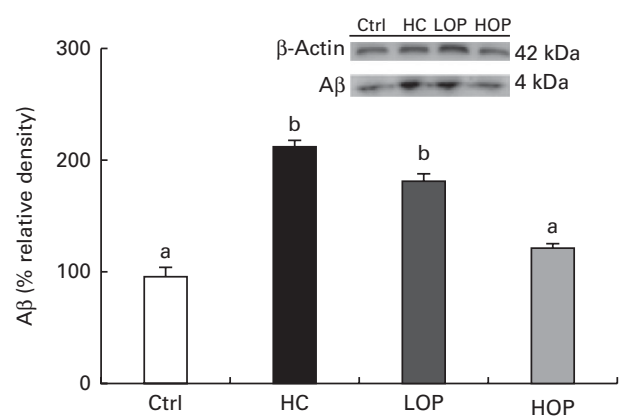

(b)

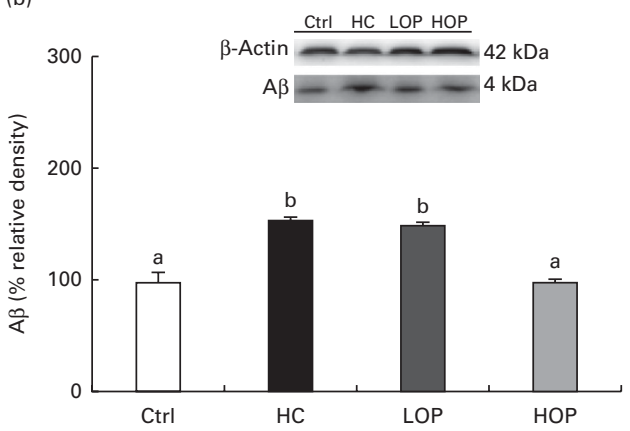

Fig. 9. Expression of $\beta$-amyloid $(A \beta)$ protein in $(A)$ the cortex and $(B)$ the hippocampus in mice. Values are means ( $n 3$ per group) of the percent relative density (with the control set to $100 \%$ ), with their standard errors represented by vertical bars. ${ }^{\mathrm{a}, \mathrm{b}}$ Mean values with unlike letters were significantly different $(P=0.05$; Duncan post hoc analysis). Ctrl, control; HC, high cholesterol; LOP, high cholesterol + low Oriental plum; HOP, high cholesterol + high Oriental plum.

an antioxidant to attenuate brain cholesterol oxidation. The increased Cyp46 mRNA expression might reflect an increase in its protein expression, which in turn might lead to the increased production of $24-\mathrm{OHC}^{(22)}$. Although we did not measure the protein level of Cyp46, this assumption is supported by the fact that significantly increased 24-OHC was found in the cortex and hippocampus in the HC group. It has been shown that the rate of transporting 24-OHC out of the brain is slower than that of forming 24-OHC from cholesterol; this might have caused too much 24-OHC and cholesterol in the brain in the present study. The raised 24OHC might represent a higher catalytic efficiency of Cyp46. It should be noted that this increase seemed to be higher in the hippocampus than in the cortex, even though both areas appeared to have a similar degree of Cyp46 mRNA expression. Because Cyp46 mRNA and protein expression is neuronspecific $^{(24)}$, we speculated that the difference in $24-\mathrm{OHC}$ levels between the cortex and the hippocampus after cholesterol administration might be the result of other independent pathways, for example, the regulation of Cyp46 at the posttranslation level. Further studies are warranted to elucidate this assumption. In addition, 24-OHC has been reported to prevent against the formation of amyloid ${ }^{(25)}$, which confirms the present finding of lower levels of $\mathrm{A} \beta$ in the hippocampus than in the cortex in the HC group. Although a clinical study has shown that 24-OHC was higher in the frontal cortices of $\mathrm{AD}$ patients as compared to the controls ${ }^{(26)}$, there is a lack of evidence to support a correlation between the levels of 24-OHC in the different subregions (i.e. the cortex and the hippocampus) and cognitive impairments. The increased levels of 24-OHC observed in patients with neurodegenerative disorders were more likely the consequence of blood-brain barrier damage or neuronal cell death rather than the metabolic inactivity of a large number of neuronal cells ${ }^{(27,28)}$.

Polyphenols are natural antioxidants. It has been shown that polyphenols reduce serum cholesterol by inhibiting cholesterol absorption, increasing bile flow and inhibiting the activities of 3-hydroxy-3-methylglutaryl-CoA reductase and acyl-CoA:cholesterol acyltransferase ${ }^{(29,30)}$. In addition, polyphenols are able to penetrate the blood-brain barrier and hence to protect neurons from oxidative stress ${ }^{(31)}$. In the present study, those mice that consumed diets containing plum powder had lower peripheral plasma cholesterol and brain cholesterol, which may be the reason why they performed better in the cognitive tests than those that did not consume plum-containing diets. Furthermore, the present findings demonstrated that consuming a diet with 5\% Oriental plum powder significantly decreased plasma and brain cholesterol concentrations, the mRNA expression of Cyp46, BACE1 protein, $\mathrm{A} \beta$ protein and $24-\mathrm{OHC}$ in the cortex and the hippocampus and improved the cognitive function of mice that had cognitive declines as the result of a highcholesterol diet. This suggests that a plum powder-containing diet may be beneficial for preventing hypercholesterolaemiarelated cognitive impairment. Additionally, 2 and 5\% Oriental plum powder-supplemented diets are equivalent to 32.4 and $81 \mathrm{~g}$ of plum powder for a $60 \mathrm{~kg}$ human per $\mathrm{d}$, respectively, based on the body surface area formula ${ }^{(32)}$. According to the United States Department of Agriculture food consumption database, the standard serving of dried plums is $42 \mathrm{~g}$ (five dried plums) ${ }^{(33)}$. Consequently, the dose of daily plum powder (approximately 1 or 2 servings) used in the present study was similar to the typical suggested adult intake. The larger dose (i.e. $5 \%$ plum powder supplementation) proved to be more effective in delaying hypercholesterolaemiarelated cognitive declines. Accordingly, a higher-percentage plum powder diet is theoretically consumable and more practical for further clinical studies to determine whether the same significant effect is found in human subjects with hypercholesterolaemia.

\section{Acknowledgements}

We thank Kao-Ting Lee of Sentosa Company Limited for providing technical support during the present experiment. We also thank Professor Si-Chuan Shen of National Taiwan Normal University for providing suggestions.

P.-H. K. contributed to the lab work. P.-H. K. and C.-I. L. drafted the manuscript. W.-C. C. provided advice on several aspects of the study. Y.-H. C. helped design the experiment, discussed the results and commented on the manuscript. S.-H. L. conducted the experiment, interpreted the data, supervised the research project and completed the manuscript.

The authors declare no conflicts of interest. 


\section{References}

1. Raffai RL \& Weisgraber KH (2003) Cholesterol: from heart attacks to Alzheimer's disease. J Lipid Res 44, 1423-1430.

2. Glenner GG \& Wong CW (1984) Alzheimer's disease: initial report of the purification and characterization of a novel cerebrovascular amyloid protein. Biochem Biophys Res Commun 120, 885-890.

3. Puglielli L, Tanzi RE \& Kovacs DM (2003) Alzheimer's disease: the cholesterol connection. Nat Neurosci $\mathbf{6}$, 345-351.

4. Notkola IL, Sulkava R, Pekkanen J, et al. (1998) Serum total cholesterol, apolipoprotein E $\epsilon 4$ allele, and Alzheimer's disease. Neuroepidemiology 17, 14-20.

5. Wolozin B (2001) A fluid connection: cholesterol and Abeta. Proc Natl Acad Sci U S A 98, 5371-5373.

6. Kolsch H, Lutjohann D, Tulke A, et al. (1999) The neurotoxic effect of 24-hydroxycholesterol on SH-SY5Y human neuroblastoma cells. Brain Res $\mathbf{8 1 8}, 171-175$.

7. Bjorkhem I (2002) Do oxysterols control cholesterol homeostasis? J Clin Invest 110, 725-730.

8. Schonknecht P, Lutjohann D, Pantel J, et al. (2002) Cerebrospinal fluid 24S-hydroxycholesterol is increased in patients with Alzheimer's disease compared to healthy controls. Neurosci Lett 324, 83-85.

9. Lau FC, Shukitt-Hale B \& Joseph JA (2005) The beneficial effects of fruit polyphenols on brain aging. Neurobiol Aging 26, 128-132.

10. Kao-Ting L, Yue-Hwa C, Ching-I L, et al. (2013) Consumption of oriental plums improved the cognitive performance and modulated the cerebral neurodegeneration-related protein expressions in rats with nicotinamide/streptozotocininduced diabetes. Food Nutr Sci 4, 1145-1154.

11. Boyer J \& Liu RH (2004) Apple phytochemicals and their health benefits. Nutr J 3, 5 .

12. Osada K, Suzuki T, Kawakami Y, et al. (2006) Dosedependent hypocholesterolemic actions of dietary apple polyphenol in rats fed cholesterol. Lipids 41, 133-139.

13. Lu J, Wu D-M, Zheng Z-H, et al. (2011) Troxerutin protects against high cholesterol-induced cognitive deficits in mice. Brain 134, 783-797.

14. Vorhees CV \& Williams MT (2006) Morris water maze: procedures for assessing spatial and related forms of learning and memory. Nat Protoc 1, 848-858.

15. Yagi K (1998) Simple assay for the level of total lipid peroxides in serum or plasma. Methods Mol Biol 108, 101-106.

16. Folch J, Lees M \& Sloane Stanley GH (1957) A simple method for the isolation and purification of total lipides from animal tissues. J Biol Chem 226, 497-509.

17. Thirumangalakudi L, Prakasam A, Zhang R, et al. (2008) High cholesterol-induced neuroinflammation and amyloid precursor protein processing correlate with loss of working memory in mice. J Neurochem 106, 475-485.
18. Refolo LM, Malester B, LaFrancois J, et al. (2000) Hypercholesterolemia accelerates the Alzheimer's amyloid pathology in a transgenic mouse model. Neurobiol Dis 7, 321-331.

19. Bouillot C, Prochiantz A, Rougon G, et al. (1996) Axonal amyloid precursor protein expressed by neurons in vitro is present in a membrane fraction with caveolae-like properties. J Biol Chem 271, 7640-7644.

20. Reiss AB \& Voloshyna I (2012) Regulation of cerebral cholesterol metabolism in Alzheimer disease. J Invest Med 60, 576-582.

21. Umeda T, Tomiyama T, Kitajima E, et al. (2012) Hypercholesterolemia accelerates intraneuronal accumulation of Abeta oligomers resulting in memory impairment in Alzheimer's disease model mice. Life Sci 91, 1169-1176.

22. Ohyama Y, Meaney S, Heverin M, et al. (2006) Studies on the transcriptional regulation of cholesterol 24-hydroxylase (CYP46A1) marked insensitivity toward different regulatory axes. J Biol Chem 281, 3810-3820.

23. Aytan N, Tamtürk F, Kartal-özera N, et al. (2008) Oxidative stress related changes in the brain of hypercholesterolemic rabbits. Biofactors 33, 225-236.

24. Russell DW, Halford RW, Ramirez DM, et al. (2009) Cholesterol 24-hydroxylase: an enzyme of cholesterol turnover in the brain. Annu Rev Biochem 78, 1017-1040.

25. Björkhem I (2006) Crossing the barrier: oxysterols as cholesterol transporters and metabolic modulators in the brain. J Intern Med 260, 493-508.

26. Heverin M, Bogdanovic N, Lütjohann D, et al. (2004) Changes in the levels of cerebral and extracerebral sterols in the brain of patients with Alzheimer's disease. J Lipid Res 45, 186-193.

27. Leoni V, Masterman T, Mousavi FS, et al. (2004) Diagnostic use of cerebral and extracerebral oxysterols. Clin Chem Lab Med 42, 186-191.

28. Leoni V, Masterman T, Patel P, et al. (2003) Side chain oxidized oxysterols in cerebrospinal fluid and the integrity of blood-brain and blood-cerebrospinal fluid barriers. J Lipid Res 44, 793-799.

29. Krecman V, Skottova N, Walterova D, et al. (1998) Silymarin inhibits the development of diet-induced hypercholesterolemia in rats. Planta Med 64, 138-142.

30. Gorinstein S, Leontowicz H, Leontowicz M, et al. (2005) Changes in plasma lipid and antioxidant activity in rats as a result of naringin and red grapefruit supplementation. J Agric Food Chem 53, 3223-3228.

31. Williams RJ \& Spencer JP (2012) Flavonoids, cognition, and dementia: actions, mechanisms, and potential therapeutic utility for Alzheimer disease. Free Radic Biol Med 52, 35- 45.

32. Reagan-Shaw S, Nihal M \& Ahmad N (2008) Dose translation from animal to human studies revisited. FASEB $J$ 22, 659-661.

33. Stacewicz-Sapuntzakis M (2013) Dried plums and their products: composition and health effects - an updated review. Crit Rev Food Sci Nutr 53, 1277-1302. 